CLINICAL STUDY

\title{
Incidence of hyperthyroidism in Stockholm, Sweden, 2003-2005
}

Mirna Abraham-Nordling, Ove Törring ${ }^{1,2}$, Mikael Lantz ${ }^{3}$, Bengt Hallengren ${ }^{3}$, Hans Ohrling ${ }^{1,2}$, Göran Lundell ${ }^{4}$, Jan Calissendorff ${ }^{5}$, Gun Jörneskog ${ }^{6,7}$ and Göran Wallin

Division of Surgery, Department of Molecular Medicine and Surgery, Karolinska Institutet, Karolinska University Hospital, SE-17176 Stockholm, Sweden, ${ }^{1}$ Department of Clinical Research and Education Sodersjukhuset, Karolinska Institutet, Sodersjukhuset, SE-11883 Stockholm, Sweden, ${ }^{2}$ Division of Endocrinology, Department of Internal Medicine, Sodersjukhuset, SE-11883 Stockholm, Sweden, ${ }^{3}$ Department of Endocrinology, Malmö University Hospital, SE-21420 Malmö, Sweden, ${ }^{4}$ Department of Oncology, Karolinska University Hospital, SE-17176 Solna, Sweden, ${ }^{5}$ Department of Endocrinology, Metabolism and Diabetes, Karolinska University Hospital, Karolinska Institutet, SE-17176 Stockholm, Sweden, ${ }^{6}$ Department of Clinical Sciences, Karolinska Institutet, SE-17177 Stockholm, Sweden and ${ }^{7}$ Department of Medicine, Danderyd Hospital, SE-18288 Stockholm, Sweden

(Correspondence should be addressed to M Abraham-Nordling; Email: mirna.nordling@ds.se)

\begin{abstract}
Objectives: To investigate the incidence of hyperthyroidism in Stockholm County, in those patients who were diagnosed with hyperthyroidism for the first time during the years 2003-2005.

Design: All new cases of hyperthyroidism $\geq 18$ years of age were prospectively registered to calculate the total incidence of hyperthyroidism, as well as the incidence of the subgroups: Graves' disease (GD), toxic multinodular goitre and solitary toxic adenoma (STA). Eight specialized units/hospitals in Stockholm County participated in the registration. The participating physicians were all specialists in medical endocrinology, oncology, nuclear medicine or surgery.

Results: During a 3-year period, 1431 new patients of hyperthyroidism were diagnosed in a well-defined adult population ( $>18$ years of age) of in average 1457036 inhabitants. This corresponds to a mean annual incidence of hyperthyroidism of 32.7/100 000. The incidence of GD was 24.5/100 000 per year, toxic nodular goitre was 3.3/100 000 per year and STA was 4.9/100 000 per year.

Conclusions: The total incidence of hyperthyroidism in Stockholm County was found to be 32.7/100 000 per year, of which $75 \%$ had GD. There were a higher percentage of smokers among the patients with hyperthyroidism compared with the overall population in Stockholm, but no difference in the frequency of smoking between patients with GD and toxic nodular goitre.
\end{abstract}

European Journal of Endocrinology 158 823-827

\section{Introduction}

Thyrotoxicosis is caused by excessive quantities of circulating thyroid hormones. The clinical presentation depends on how various organs are affected by these hormonal levels at tissue level. There are three main types of hyperthyroidism caused by increased thyroid hormone production: Graves' disease (GD), toxic multinodular goitre (TMNG) and solitary toxic adenoma (STA). GD accounts for most of the patients with hyperthyroidism and the highest risk for onset is between 40 and 60 years of age (1). The incidence in different areas depends upon iodine intake. If there is a low supply of iodine, the thyroid compensates by a number of mechanisms in an attempt to maintain sufficient thyroid hormone production. The rate of hyperthyroidism varies in different countries and affects $\sim 2 \%$ of the women and $0.2 \%$ of the men $(2,3)$.

The incidence of hyperthyroidism has been reported in Malmö, Sweden, during the years 1970-1974 and 1988$1990(4,5)$. The incidence was shown to increase from 25.8 in the early study to 43.0 per 100000 inhabitants per year in the last study. The cause of the increased incidence was unknown but was probably influenced by more sensitive diagnostic tools and changes in smoking habits. Another study in a northern inland area of Sweden calculated an incidence of 34.7/100 000 per year. The incidence of toxic nodular goitre decreased between the first and second 5-year period of the study during the years 1975-1984 (6).

The aim of this study was to determine the total and age-specific incidence of hyperthyroidism and to identify the subgroups (GD, TMNG and STA) during a period of 3 years (2003-2005) in Stockholm County.

\section{Materials and methods}

\section{Study population}

The mean number of residents in the region of Stockholm County was 1867995 inhabitants during the study period. We excluded all persons who were 
under 18 years of age (22\%). The average size of the study population during this 3-year period therefore was 1457036 inhabitants.

All patients had, because of hyperthyroidism, been referred to eight specialized units/hospitals in Stockholm County, which participated in the registration. In Stockholm, the treatment of hyperthyroidism is mainly decided and initiated by a specialist physician in those units. The physicians, who were participating in the registration, were all specialists in medical endocrinology, oncology, nuclear medicine or surgery. All patients from the Stockholm population newly diagnosed with hyperthyroidism (GD, TMNG and STA) for the first time during the 3-year period ranging from 1 January 2003 to 31 December 2005 were included in the study. A letter was also sent to all primary healthcare centres in Stockholm regarding possible patients with hyperthyroidism, who had not been referred to a specialized unit, to be certain that all patients were included in the study.

The patients were identified by having symptoms and/or signs of hyperthyroidism in combination with elevated levels of one or several of the following thyroid hormones: total serum thyroxin $\left(\mathrm{T}_{4}\right)$, free $\mathrm{T}_{4}\left(\mathrm{fT}_{4}\right)$, total serum tri-iodothyronine $\left(\mathrm{T}_{3}\right)$, free $\mathrm{T}_{3}\left(\mathrm{fT}_{3}\right)$ and suppressed TSH $(<0.02 \mathrm{mU} / \mathrm{l})$. Autoimmunity was screened by measuring antibodies to thyrotrophin receptor antibody (TRab) and thyroid peroxidase antibody.

All patients who had laboratory-confirmed hyperthyroidism and diffuse goitre, positive TRab and/or a verifying thyroid scintigraphy showing diffuse pattern of isotope uptake were diagnosed as GD. All patients with laboratory-confirmed hyperthyroidism and single or multinodular goitre, and absence of TRab were classified as STA and TMNG. This diagnosis was confirmed by scintigraphy showing local increased isotope uptake with the suppression of normal thyroid tissue in the remaining part of the gland in doubtful cases. Exclusion criteria were patients with past or current history of hyperthyroidism, with destructive thyroiditis or hyperthyroidism secondary to pharmacological treatment with amiodarone or interferon.

\section{Study design and data collection}

We performed a prospective registration of patients diagnosed as hyperthyroidic during the years 20032005 in Stockholm County, Sweden. Before the study was started, all the hospital specialists were notified and a questionnaire circulated to optimize its content. The registration of patients was made only at the first visit when the patient met a specialist who confirmed the diagnosis.

The following data were registered: age, sex, ethnic origin (country of birth), type of hyperthyroidism (GD, TMNG or STA), amiodarone treatment, smoking habits, occurrence of endocrine ophthalmopathy and pretibial myxoedema. A blood sample was drawn and laboratory tests were evaluated for current thyroid hormonal levels. A diagnostic thyroid scintigraphy and a 24-h ${ }^{131}$ I uptake test were performed in selected cases. For each patient the initially planned treatment was registered.

The study was approved by the local ethic committee at Karolinska Institutet (KI: 02-520).

\section{Statistical analysis}

The incidence rate was calculated using the age- and sex-matched population. The Statistica 7.1, StatSoft Inc., Tulsa, OK, USA was used for all computer analyses.

\section{Results}

\section{Total incidence}

In this 3-year period, 1431 patients with hyperthyroidism were diagnosed, in which 1130 were females and 291 males, giving a female to male ratio of 3.9:1. The mean annual incidence of hyperthyroidism was 32.7 per 100000 (Table 1). The annual incidence for females was 50.8 per 100000 and for males was 13.6 per 100000 .

\section{Hyperthyroidism - subgroup incidence}

GD occurred in 1071 patients corresponding to an annual incidence of 24.5 per 100 000. TMNG occurred in 146 patients (incidence 3.3 per 100 000) and STA in 214 patients (incidence 4.9 per 100 000; Table 1).

\section{Age-specific incidence}

The majority of the patients were between 40 and 80 years of age. The highest incidences for female patients were seen in the age groups of 50-59 (66.0/100 000 per year) and 70-79 years (68.3/100 000 per year). For male patients, the incidence had an increasing trend with age, with the highest peak over 90 years of age (31.9/100 000 per year) $(n=3$ patients; Fig. 1$)$.

The highest annual incidence of GD was in the middleaged patients; the peak incidence (29.4 per 100000 per year) was seen in the group of 30- to 39-year old (Fig. 2).

Table 1 Number of patients with different subgroups of hyperthyroidism in Stockholm 2003-2005. Percentage is given in brackets.

\begin{tabular}{lllll}
\hline & Total & GD & TMNG & STA \\
\hline $\begin{array}{l}\text { Total number of } \\
\text { patients }\end{array}$ & 1431 & $1071(75 \%)$ & $146(10 \%)$ & $214(15 \%)$ \\
$\begin{array}{l}\text { Mean incidence } \\
\text { (/100 000 per year) }\end{array}$ & 32.7 & 24.5 & 3.3 & 4.9 \\
$\begin{array}{l}\text { Female } \\
\text { Male }\end{array}$ & 1130 & $820(73 \%)$ & $130(12 \%)$ & $180(16 \%)$ \\
$\begin{array}{l}\text { Ratio of females:- } \\
\text { males }\end{array}$ & 291 & $241(83 \%)$ & $16(5 \%)$ & $34(12 \%)$ \\
& & $3.4: 1$ & $8.1: 1$ & $5.3: 1$ \\
\end{tabular}




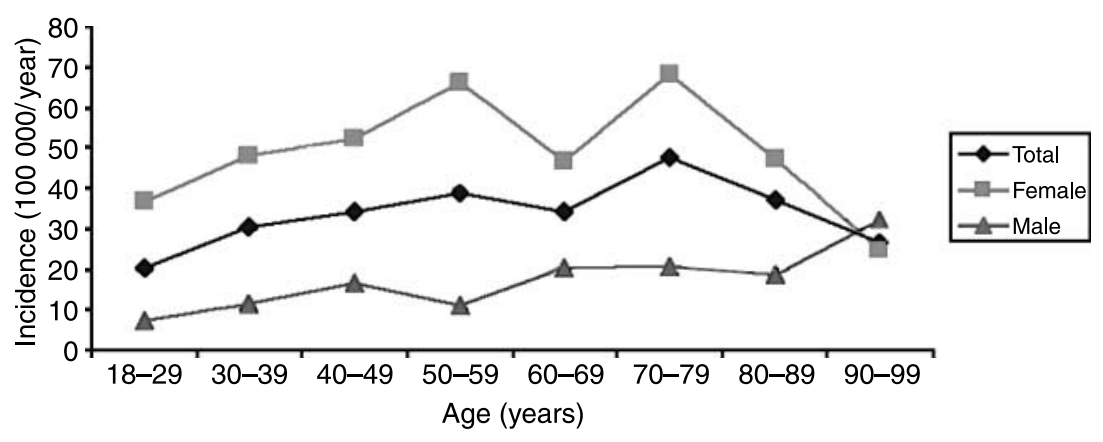

Figure 1 Annual incidence of hyperthyroidism in Stockholm 2003-2005 divided on the basis of age and sex.
The annual incidence for TMNG and STA showed an increasing incidence with age. For TMNG the peak was at the age group of 70-79 years (12.5 per 100000 per year) and for STA the peak was at the age group of 70-79 years (17.1 per 100000 per year; Fig. 2).

Among the 1071 Graves' patients, there were 820 females and the majority of the patients were between the ages 30 and 60 years. With increasing age, the incidence of GD is decreasing in females (Fig. 3).

\section{Smoking habits}

An exploratory question about smoking habits was answered in 1274 cases. The patients were equally distributed in the non-smoking group in the three types of hyperthyroidism: GD (42\%), TMNG (45\%) and STA $(37 \%)$. In the (previous smokers) ex-smoking group, a trend to higher frequency was seen in the STA (33\%) and TMNG $(26 \%)$ compared with GD with $22 \%$. The group of smokers were equally distributed among GD (24\%), TMNG (21\%) and STA (24\%; Table 2).

Among all females and males, there were 43 and $35 \%$ in the non-smoking group and 22 and $31 \%$ in the ex-smoking group, and there were $24 \%$ each in the smoking group respectively. For both genders this question was not answered in $11 \%$.

\section{Discussion}

The Stockholm County is a large area with eight specialized main hospitals where patients with hyperthyroidism are diagnosed and treated. Despite the fact that the questionnaire was circulated among the hospital specialists before the study was initiated to optimize its content, there could still be some patients who were not included in the study. To minimize this error as much as possible, we have analyzed lists of all patients with a diagnostic code for hyperthyroidism and thyrotoxicosis, who visited the included hospitals during the study period to be certain that as few patients as possible were not registered. To further minimize this possible problem, a letter was sent to all primary healthcare centres to report whether they have treated any patients with hyperthyroidism during this period. There were no additional cases reported from the primary healthcare centres. In the present study, the incidence was reported for the adult population. If children $<18$ years were included, the incidence number might be lower since hyperthyroidism is more uncommon among children.

The incidence of hyperthyroidism in Stockholm County is 32.7 per 100000 per year (Table 1). It is comparable with other studies where the incidence varies between 23 and 93/100 000 per year (4-12).

The causes of hyperthyroidism are influenced by the endemic iodine exposure. GD has been shown to be more common among the new cases of hyperthyroidism in Iceland where the iodine intake is high-normal $(300 \mu \mathrm{g} /$ day) whereas multinodular or uninodular goitre causes over half of the new cases in an area of low iodine intake (40-70 $\mu \mathrm{g} /$ day) such as Jutland, Denmark $(7,13)$. Iodine intake also affects the total incidence of hyperthyroidism since Iceland had lower

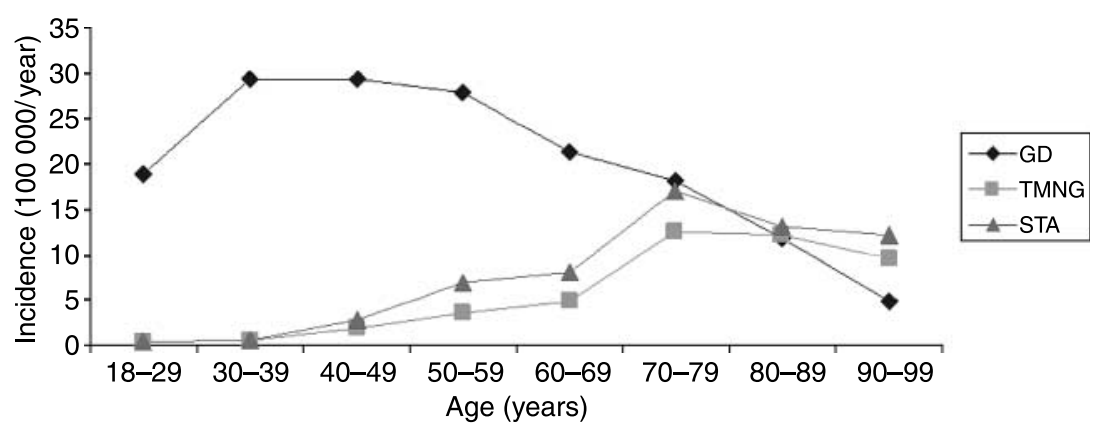

Figure 2 Annual incidence of subgroups of hyperthyroidism divided on the basis of different ages in Stockholm 2003-2005. 


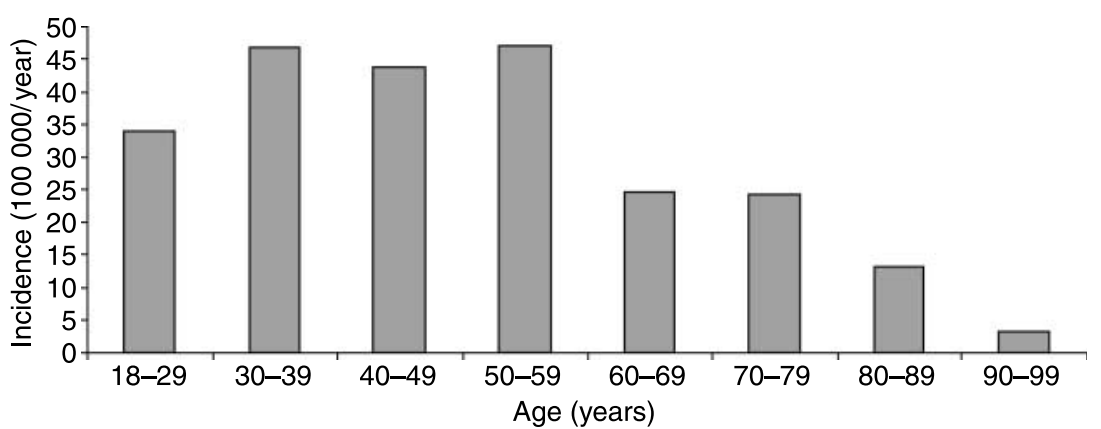

Figure 3 Age-specific incidence of Graves' disease in women in Stockholm 2003-2005. incidence compared with Denmark. Another aspect of endemic iodine status is the changed prevalence in thyroid diseases, which iodine supplementation creates when general iodination programmes are started in iodine-deficient areas. In this situation, some individuals with a latent Graves' hyperthyroidism may aggravate to hyperthyroidism. Also patients with nontoxic or autonomous nodular goitre may progress to a toxic state $(8,14-16)$.

It is therefore important to consider the iodine status in Sweden in relation to the present study. In Sweden, endemic goitre was found in many regions at the beginning of the 20th century. With the introduction of table salt iodization in $1936(10 \mathrm{mg} / \mathrm{kg})$, endemic goitre became less frequent. In 1966, the table salt iodization was increased from 10 to $50 \mathrm{mg} / \mathrm{kg}$ because some areas still had a high incidence of goitre. Our iodine supplementation programme has thus been established for more than four decades and a recent national survey of iodine intake in Sweden including the Stockholm area indicates a generally sufficient iodine intake (unpublished observation). We therefore consider the incidences of hyperthyroidism in the present study to be observed during a stable and sufficient iodine intake situation.

The discrepancy in incidence in the current investigation compared with the previous Swedish studies is due to the differences in toxic nodular goitre since the incidence of GD of 24.5/100 000 per year is in close agreement with the earlier reports (17-22/100 000 per year) (4-6). GD accounted for $75 \%$ of the total hyperthyroidism patients in the present study and it is known that this disease is calculated to be $60-80 \%$ of the total number of patients with hyperthyroidism, depending on regional factors, especially iodine intake (9).

The age-specific incidence of GD was, as suspected, the highest among the young patients (Fig. 2). The STA incidence was 4.9/100 000 and for TMNG it was 3.3/ 100000 per year. TMNG had the lowest incidence among the three types of hyperthyroidism. Previously, the incidence of these two types has been reported to be 5.4-16.0/100 000 per year for TMNG and 2.7-4.8/ 100000 per year for STA $(4,5)$. The low incidence of TMNG in the current and the other previous Swedish studies are probably due to the fact that these types are more common in areas with iodine deficiency (17). It is therefore tempting to speculate whether the lower incidence of TMNG in the present study is related to the iodination programme. For example, is it possible that there has been a short-term 'flare' of thyrotoxicosis in TMNGs during the 1970s and 1980s, as the $50 \mathrm{mg}$ iodine/per kilo table salt became more widely used after its introduction in 1966, and that this boom of TMNG has settled? Moreover, did the iodination programme result in less frequent development of nodular goitre in subjects who were young during these decades and who now would be in their middle or old age and thereby contribute to a decreased incidence of TMNG in the present study? Meanwhile have the middle- or old-aged subjects with nodular goitre at that time and candidates for TMNG at present died due to higher age-related mortality? The age-related increase in TMNG in the present study could support such hypotheses.

However, the present study was not designed to enlighten this perspective. The present incidence study was performed in Stockholm while previous data on TMNG have been collected in southern Sweden (Malmo) in late 1990s $(4,5)$. Since no previous measuring point is available, we cannot confirm that the incidence of TMNG has decreased in the Stockholm region. In addition, there are region-related possible confounders such as socio-economic factors, smoking and emigration patterns.

The total incidence rate for females is 50.8/100 000 per year and for males 13.6/100 000 per year giving a ratio of 4:1 female: male. It is in accordance with the ratios from previous studies (female:male 5.6:1-6.6:1) $(4,18)$.

Table 2 Smoking habits of hyperthyroidism in Stockholm 2003-2005.

\begin{tabular}{lcccc}
\hline & $\begin{array}{c}\text { Total } \\
\boldsymbol{n}=\mathbf{1 4 3 1} \\
(\%)\end{array}$ & $\begin{array}{c}\text { GD } \\
\boldsymbol{n}=\mathbf{1 0 7 1} \\
(\%)\end{array}$ & $\begin{array}{c}\text { TMNG } \\
\boldsymbol{n}=\mathbf{1 4 6} \\
(\%)\end{array}$ & $\begin{array}{c}\text { STA } \\
\boldsymbol{n}=\mathbf{2 1 4} \\
(\%)\end{array}$ \\
\hline Non-smokers & $595(42 \%)$ & $449(42 \%)$ & $66(45 \%)$ & $80(37 \%)$ \\
Ex-smokers & $340(24 \%)$ & $232(22 \%)$ & $38(26 \%)$ & $70(33 \%)$ \\
Smokers & $339(24 \%)$ & $257(24 \%)$ & $31(21 \%)$ & $51(24 \%)$ \\
Unknown & $157(11 \%)$ & $133(12 \%)$ & $11(8 \%)$ & $13(6 \%)$ \\
\hline
\end{tabular}


It is well established that smoking habits increase the risk to develop GD as well as the relapse rate for medically treated patients. The mechanism behind this is not understood $(19,20)$. In this exploratory study of smoking habits, smoking did not contribute to the correlation within the subgroups, as the smokers were divided equally among the three types of hyperthyroidism (Table 2). We had expected to see a higher rate of smokers in the GD group. The explanation behind the equal number of smokers in the subgroups can be that smoking is only one of the many environmental factors that combined with genetic factors, can trigger the disease. Comparing the smoking habits of the investigated patients' hyperthyroidism with the overall adult population in Stockholm, we found that smoking was less common among the overall adult population in Stockholm for the year 2005 (total $16 \%$, females $17 \%$ and males $14 \%$ ). This shows that smoking is more correlated to hyperthyroidism and less to the subgroups of hyperthyroidism.

In conclusion, this study has for the first time defined the incidence of hyperthyroidism in a large study population in Stockholm County, Sweden. The majority of hyperthyroidism is due to GD. Our results are important for future research and from healtheconomic perspectives.

\section{Acknowledgements}

The authors wish to thank the Hyperthyroidism Incidence Study Group in Stockholm (Hyperthyroidism Incidence Study Group in Stockholm comprises: Dr H Falhammar, Dr J Hoffstedt, Dr Y Pernow, Dr E Moberg, Dr B Freyschuss, Dr M Degerblad, Dr H Wagner, Dr L Juntti-Berggren, Dr M Alvarsson, K Byström Division of Endocrinology, Karolinska University Hospital. Dr C Hilding, Dr A-L Hjelm, Dr Karimi, Dr G Iliescu, Dr M Frank, Division of Oncology, Karolinska University Hospital. Dr O Mattson, Dr Volpe, Dr S Röjdmark, Dr J Friberg, Dr P Curman, Dr Nathanson, J Friberg, Dr T Nyström Division of Endocrinology, Sodersjukhuset. Dr M V Heijne, Division of Medicin, Danderyd Hospital. Dr E Fernqvist-Forbes, Division of Diabetes and Endocrinology, Nacka Hospital. Dr E Toft, Division of Diabetes, Ersta Hospital. Dr M Palmer, Division of Endocrinology, S: t Görans Hospital), all patients and staff who made this survey possible. The study was supported by the Swedish Research Council and the Karolinska Institutet.

\section{References}

1 Weetman AP. Graves' disease. New England Journal of Medicine $20003431236-1248$.

2 Franklyn J. Thyrotoxicosis. Clinical Medicine 20033 11-15.
3 Tunbridge WM, Evered DC, Hall R, Appleton D, Brewis M, Clark F, Evans JG, Young E, Bird T \& Smith PA. The spectrum of thyroid disease in a community: the Whickham survey. Clinical Endocrinology 1977 7 481-493.

4 Berglund J, Christensen SB \& Hallengren B. Total and age-specific incidence of Graves' thyrotoxicosis, toxic nodular goitre and solitary toxic adenoma in Malmo 1970-74. Journal of Internal Medicine 1990227 137-141.

5 Berglund J, Ericsson UB \& Hallengren B. Increased incidence of thyrotoxicosis in Malmo during the years 1988-1990 as compared to the years 1970-1974. Journal of Internal Medicine $199623957-62$.

6 Lundgren E \& Borup Christensen S. Decreasing incidence of thyrotoxicosis in an endemic goitre inland area of Sweden. Clinical Endocrinology 199033 133-138.

7 Haraldsson A, Gudmundsson ST, Larusson G \& Sigurdsson G. Thyrotoxicosis in Iceland 1980-1982. An epidemiological survey. Acta Medica Scandinavica $1985217253-258$.

8 Laurberg P, Bulow Pedersen I, Pedersen KM \& Vestergaard H. Low incidence rate of overt hypothyroidism compared with hyperthyroidism in an area with moderately low iodine intake. Thyroid 19999 33-38.

9 Vanderpump MP, Tunbridge WM, French JM, Appleton D, Bates D, Clark F, Grimley Evans J, Hasan DM, Rodgers H, Tunbridge F \& Young ET. The incidence of thyroid disorders in the community: a twenty-year follow-up of the Whickham Survey. Clinical Endocrinology 199543 55-68.

10 Knudsen N, Bulow I, Jorgensen T, Laurberg P, Ovesen L \& Perrild H. Comparative study of thyroid function and types of thyroid dysfunction in two areas in Denmark with slightly different iodine status. European Journal of Endocrinology $2000 \mathbf{1 4 3}$ 485-491.

11 Brownlie BE \& Wells JE. The epidemiology of thyrotoxicosis in New Zealand: incidence and geographical distribution in north Canterbury, 1983-1985. Clinical Endocrinology 199033 249-259.

12 Leese GP, Flynn RV, Jung RT, Macdonald TM, Murphy MJ \& Morris AD. Increasing prevalence and incidence of thyroid disease in Tayside, Scotland: the Thyroid Epidemiology Audit and Research Study (TEARS). Clinical Endocrinology 200868 311-316.

13 Laurberg P, Bulow Pedersen I, Knudsen N, Ovesen L \& Andersen S. Environmental iodine intake affects the type of nonmalignant thyroid disease. Thyroid 200111 457-469.

14 Connolly RJ. An increase in thyrotoxicosis in southern Tasmania after an increase in dietary iodine. Medical Journal of Australia $197111268-1271$.

15 Bulow Pedersen I, Laurberg P, Knudsen N, Jorgensen T, Perrild H, Ovesen L \& Rasmussen LB. Increase in incidence of hyperthyroidism predominantly occurs in young people after iodine fortification of salt in Denmark. Journal of Clinical Endocrinology and Metabolism 200691 3830-3834.

16 Laurberg P, Andersen S, Pedersen IB, Ovesen L \& Knudsen N. Humic substances in drinking water and the epidemiology of thyroid disease. Biofactors 200319 145-153.

17 Roti E \& Uberti ED. Iodine excess and hyperthyroidism. Thyroid $200111493-500$.

18 Flynn RW, Macdonald TM, Morris AD, Jung RT \& Leese GP. The thyroid epidemiology, audit, and research study: thyroid dysfunction in the general population. Journal of Clinical Endocrinology and Metabolism 200489 3879-3884.

19 Cooper DS. Hyperthyroidism. Lancet $2003362459-468$.

20 Vestergaard P, Rejnmark L, Weeke J, Hoeck HC, Nielsen HK, Rungby J, Laurberg P \& Mosekilde L. Smoking as a risk factor for Graves' disease, toxic nodular goiter, and autoimmune hypothyroidism. Thyroid 200212 69-75.

Received 18 February 2008

Accepted 28 February 2008 